\title{
EDIFICATE
}

I Congreso de Escuelas de Edificación y Arquitectura Técnica de España València, 4 y 5 de noviembre de 2021

Escuela Técnica Superior de Ingeniería de Edificación

Universitat Politècnica de València

Doi: https://doi.org/10.4995/EDIFICATE2021.2021.13537

\section{Metodología y recursos didácticos claves en el proceso de enseñanza-aprendizaje de las asignaturas de Construcción II y III del Grado de Arquitectura Técnica.}

\section{Methodology and key didactic resources used in teaching- learning process in the subjects of Construction II and III on Bachelor's Degree in Technical Architecture.}

\footnotetext{
José Manuel Gandía-Romeroa, Milagro lborra Lucas ${ }^{b}$, Ana Martínez lbernón ${ }^{c}$

a Universitat Politècnica de València, Depto. de Construcciones Arquitectónicas, joganro@csa.upv.es

b Universitat Politècnica de València, Depto. de Construcciones Arquitectónicas, miborra1@csa.upv.es b Universitat Politècnica de València (IDM), anmarib@arqt.upv.es
}

\begin{abstract}
Construction II (Flat roofs and sloped roofs) and Construction III (Facades, partitions and claddings) are two mandatoy subjects of second-year courses. The new European Higher Education Area and the competence-based learning approach required a global and integrated approach. This paper describes key methodological aspects used in both subjects and exposes those didactic resources considered essential to achieve the learning outcomes and the competences defined in the teaching guide. In addition, promoting the acquisition of transversal competences has been a key factor in the design of activities and for the selection of teaching resources. At the end of the work, the student's perception of the usefulness of the resources used is analyzed.
\end{abstract}

Keywords: Construction, resources, didactic metodology competences.

\footnotetext{
Resumen

Las asignaturas de Construcción II (Cubiertas planas y cubiertas inclinadas) y de Construcción III (Fachadas, particiones y revestimientos) son dos asignaturas obligatorias de segundo curso. El nuevo espacio europeo para la educación superior y el aprendizaje basado en competencias requirieron de un enfoque global e integrado. En el presente trabajo se describen aspectos metodológicos y se exponen aquellos recursos didácticos considerados
} 
Metodología y recursos didácticos claves el proceso de enseñanza-aprendizaje de las asignaturas de

Construcción II y III del Grado de Arq. Técnica - Methodology and key didactic resources used in teaching-learning process in the subjects of C II and III on Bachelor's Degree in Technical Architecture.

imprescindibles para alcanzar los resultados de aprendizaje $y$ las competencias definidas en la guía docente. El enfoque de la asignatura hacia la adquisición de competencias ha sido clave en el diseño de actividades y en la selección de los recursos. Al final del trabajo se analiza la percepción del alumno sobre la utilidad de los recursos empleados.

Palabras clave: Construcción, recursos, metodología didáctica, competencias. 


\section{Introducción}

El nuevo Espacio Europeo de Educación Superior (EEES) generó profundos cambios metodológicos en el sistema universitario (Benito, 2005). Se hizo necesaria una reformulación de los métodos de enseñanza-aprendizaje, pasando del "saber" y "aprender" al "saber hacer" y "aprender a aprender".

Actualmente, los resultados de aprendizaje no los entendemos como elementos aislados, deben además fomentar la adquisición de competencias. La competencia se puede definir como una capacidad para movilizar diversos recursos cognitivos para hacer frente a un tipo de situaciones (Perrenoud, 2007). Éstas, deben permitir al alumnado abordar con éxito los restos futuros desde una perspectiva profesional y personal (Goñi Zabala, 2005; Poumay, 2017). Se pasa, por tanto, de un modelo basado, casi exclusivamente en contenidos, a otro donde se hace necesario un enfoque global desde el punto de vista metodológico que promueva la adquisición de competencias profesionales y transversales. En la Universitat Politècnica de València (UPV) se consideran claves estas competencias $y$, es por ello que, son evaluadas y reflejadas en los expedientes académicos.

Por tanto, debe de darse importancia no solo a lo que se enseña sino también a cómo lo enseñamos. Una de las claves del nuevo modelo de educación es el método docente, lo que nos obliga como profesores a tener una visión global. Se debe guiar al alumnado y abrirle nuevos caminos, adquiriendo así la labor tutorial y de acompañamiento adquiere un gran valor. Por otro lado, al alumnado se le debe exigir una actitud claramente activa y una implicación decidida y responsable en su propia formación.

En la UPV, ya en el año 2002 se creó el Proyecto EUROPA que tenía como objetivos favorecer sinergias entre cada Centro y los Departamentos que imparten docencia en él, mejorar la cualificación del profesorado e incentivar la implantación de nuevos métodos de enseñanza y evaluación. Se hacía una propuesta de un nuevo método docente que suponía un cambio en la actividad del profesorado. Al diseñar la metodología en las asignaturas de CII y III también se tuvieron en cuenta las directrices del Plan de Acciones para la Convergencia Europea (PACE) y las de Plan Estratégico 2007-2014. En este último plan y dentro del primer eje estratégico, como objetivo I.3 se indicaba la necesidad de mejorar el rendimiento académico de los estudiantes, desarrollando algunas líneas estratégicas y planes de acción orientadas a la adecuación de las metodologías como el "Método Activa" que buscaba potenciar el uso de las metodologías activas que mejorasen la capacidad de aprendizaje y el rendimiento académico de los estudiantes y la "Docencia en Red" que buscaba desarrollar una completa oferta formativa con metodología específica TIC (cursos on-line, ejercicios prácticos, foros de aprendizaje, blogs, documentación bibliográfica,...). Finalmente, indicar también que en el Plan estratégico UPV 2015-2020, uno de los retos estratégicos es "Ser un referente en formación de calidad orientada a las necesidades de la sociedad y tiene como objetivo... avanzar hacia modelos de formación que logren que sus estudiantes adquieran las competencias necesarias para poder tener una adecuada inserción laboral. Esta formación debe verse desde una perspectiva amplia, ligada al ciclo formativo 
Metodología y recursos didácticos claves el proceso de enseñanza-aprendizaje de las asignaturas de

Construcción II y III del Grado de Arq. Técnica - Methodology and key didactic resources used in

teaching-learning process in the subjects of C II and III on Bachelor's Degree in Technical Architecture.

integral de las personas, que abarca tanto el grado como el posgrado, lo que implica un esfuerzo continuado en la renovación y mejora de la metodología".

Por todo lo expuesto, desde el punto de vista metodológico la organización e integración de los recursos didácticos en las asignaturas de Cll y CIII ha sido para los profesores un aspecto fundamental para satisfacer los resultados de aprendizaje fomentando que este aprendizaje sea significativo y que favorezca también la adquisición de competencias. El análisis de la percepción del alumnado sobre la metodología empleada y la adecuación de los recursos es clave de cara a establecer futuras acciones.

\section{Objetivos}

El objetivo principal del presente trabajo es analizar la percepción que tienen actualmente los alumnos del empleo de los recursos didácticos en las asignaturas de Cll y CIII para poder establecer futuras estrategias de mejora y/o consolidación.

Para poder contextualizar dicho análisis se han establecido los siguientes objetivos operativos: (i) Describir de forma general la organización y metodología empleada actualmente en las asignaturas de Cll y CII, (ii) identificar los recursos didácticos empleados durante el proceso de enseñanza aprendizaje y (iii) analizar las encuestas de los alumnos.

\section{Las asignaturas de Cll y CIII del grado de Arquitectura Técnica en la UPV}

\subsection{Enfoque, competencias y contenidos en las asignaturas de Construcción II y III}

Antes de comenzar a describir la metodología se considera interesante dar un enfoque general de las asignaturas en el grado de Arquitectura Técnica (GAT). Las asignaturas de Construcción II (cubiertas planas e inclinadas) y III (fachadas, particiones y revestimientos), tienen asignados 4,5 ECTS cada una y son asignaturas obligatorias de segundo curso que tienen como objetivos comunes hacer que los alumnos consoliden la aplicación de los principios constructivos estudiados en la asignatura de Construcción I (Introducción a la construcción) y sobre todo que profundicen en los sistemas específicos de las asignaturas de Cll y III, esto es, identificando los materiales que componen los sistemas y las relaciones entre los diferentes elementos constructivos. Se pretende que el alumnado sea capaz de evaluar y concretar las soluciones, secuencias constructivas y puestas en obra. Se potencian las capacidades de análisis y el uso de la expresión gráfica como medio eficaz para comunicar ideas. El análisis de la normativa y la adecuación de las soluciones es otro aspecto que tiene mucha importancia. En definitiva, las asignaturas aportan el conocimiento necesario para resolver de forma correcta las cubiertas, fachadas, particiones y revestimientos, teniendo en cuenta la problemática de cada una de las tipologías y siendo capaz de tomar, con conocimiento técnico, las decisiones adecuadas para su correcta ejecución. Tanto ClI como CIII comparten profesores y metodología, Cll se imparte en el primer semestre y CIII en el segundo semestre si bien ambas asignaturas en el anterior plan de estudios estaban integradas en una asignatura anual común de tercer curso. 
La competencias definidas en la guía docente son las siguientes:

Tabla 1. Competencias específicas (CE) y transversales (CT) en Cll y CIII

\section{Competencias}

(CE01) Aptitud para identificar los elementos, sistemas y tipologías constructivas, definir su función, idoneidad relativa y compatibilidad en el proceso constructivo. Aptitud para plantear y resolver detalles constructivos.

(CE02) Conocimiento de los procedimientos específicos de control de la ejecución material de obras de edificación.

(CE03) Conocimiento de los equipos, instalaciones provisionales y medios auxiliares empleados en las obras de edificación, sus características y normativa de aplicación.

(CE04) Capacidad para evaluar las necesidades y establecer criterios para la selección de los equipos, instalaciones provisionales y medios auxiliares.

(CE05) Saber aplicar sus conocimientos a su trabajo o vocación de una forma profesional y poseer las competencias que suelen demostrarse por medio de la elaboración y defensa de argumentos y resolución de problemas dentro del área de estudio.

(CE06) Poseer habilidades de aprendizaje necesarias para emprender estudios posteriores con un alto grado de autonomía.

(CE07) Capacidad para aplicar la normativa técnica al proceso de la edificación y generar documentos de especificación técnica que determinen los procedimientos y métodos constructivos de edificios.

(CE08) Poseer y comprender conocimientos en un área de estudio que parte de la base de la educación secundaria general, y se suele encontrar a un nivel, que si bien se apoya en libros de texto avanzados, incluye también algunos aspectos que implican conocimientos procedentes de la vanguardia de su campo de estudio.

(CE09) Capacidad de transmitir información oral y escrita en la lengua nativa: ideas, problemas y soluciones a un público tanto especializado como no especializado.

(CE10) Conducta ética en ingeniería. Capacidad crítica y autocrítica. Conciencia de la necesidad de calidad y alto nivel profesional y de su aplicación al continuo perfeccionamiento.

(CE11) Conocimiento de los elementos, sistemas y tipologías constructivas, tradicionales y prefabricados empleados en la edificación y sus variedades.

(CE12) Capacidad para dictaminar sobre las causas y manifestaciones de las lesiones en los edificios, y proponer soluciones para evitar o subsanar su patología.

(CE13) Conocimiento de los procedimientos y técnicas de evaluación de la eficiencia energética de los edificios.

(CE14) Aptitud para diseñar y materializar soluciones de acondicionamiento acústico, térmico y lumínico de los mismos.

(CT02) Aplicación y pensamiento práctico

(CT03) Análisis y resolución de problemas

(CT09) Pensamiento crítico

Hay dos bloques de contenidos en CII (tejados y azoteas) y tres grandes bloques en CIII (fachadas, particiones y revestimientos). Los bloques de contenidos se estructuran en las siguientes unidades didácticas:

Tabla 2. Desglose de Unidades Didácticas en las asignaturas de Cll y CIII

\section{Construcción II}

TJ-01. Cubiertas. Introducción.

TJ-02.Tejados. Generalidades, Exigencias Básicas y Clasificación según CTE

TJ-03.Tejados de Teja. Cerámica y de Hormigón 
Metodología y recursos didácticos claves el proceso de enseñanza-aprendizaje de las asignaturas de

Construcción II y III del Grado de Arq. Técnica - Methodology and key didactic resources used in

teaching-learning process in the subjects of C II and III on Bachelor's Degree in Technical Architecture.

TJ-04. Tejados de Teja. Pizarra.

TJ-05. Tejados. Placas y Perfiles. Cubiertas Ligeras no Metálicas.

TJ-06. Tejados. Placas y Perfiles. Cubiertas Ligeras Metálicas.

AZ-07. Azoteas. Generalidades

AZ-08. Azoteas Transitables

AZ-09. Azoteas no Transitables

Construcción III

FA-01. Fachadas. Generalidades

FA-02. Fachadas. Fabrica de ladrillo

FA-03. Fachadas. Fabrica de bloque

FA-04. Fachadas. Carpintería de vidrio

FA-05. Fachadas. Muros cortina

FA-06. Fachadas. Ventiladas

FA-07. Fachadas. Otros tipos

PT-08. Particiones. Generalidades

PT-09. Particiones. Fabricas de ladrillo y bloque

PT-10. Particiones. Entramados de yeso laminado

PT-11. Particiones. Carpintería interior

$\mathrm{RV}$-12. Revestimientos verticales

RV-13. Revestimientos horizontales

\subsection{Principales actividades desarrolladas y recursos empleados en las asignaturas de Construcción II y III}

Algunos aspectos clave que se han tenido en cuenta en el diseño metodológico de las asignaturas de Construcción II y III son los siguientes:

- Diseñar actividades y procedimientos para favorecer la construcción de los aprendizajes de una forma más efectiva utilizando metodologías activas, buscando que el aprendizaje sea significativo y que se construya sobre los conocimientos previos.

- Establecer una evaluación continua del proceso, que permita obtener información y modificar o mejorar aquellos aspectos que se consideren necesarios.

- Proporcionar un feed-back en las actividades para que puedan conocer la forma de resolver las prácticas, es decir, aquellos aspectos determinantes y condicionantes de la solución.

- Explicar cuáles son los criterios de evaluación, el alumno debe conocerlos, no solo en los actos de evaluación sino en el desarrollo de prácticas y teoría.

- Favorecer el trabajo cooperativo y evaluar también aspectos relacionados con la adquisición competencias transversales.

- Motivar al alumnado con actividades que puedan resultarles más atractivas y aplicables a su futura actividad profesional. 
- Canalizar y orientar correctamente los trabajos que se plantean a lo largo del curso de modo que puedan servir como apoyo en las prácticas, en las tutorías y en el propio desarrollo de los contenidos de clase en teoría de aula.

- Fomentar el uso del laboratorio de Construcción como espacio de aprendizaje y espacio tutorial.

- Facilitar el acceso a los espacios de modo que los alumnos puedan trabajar individualmente, reflexionar y tener contacto con los recursos disponibles, ver trabajos de años anteriores seleccionados por los profesores, elaborar las maquetas...

- Incrementar los recursos materiales y de apoyo que proporcionados a los alumnos, incorporando documentación gráfica y facilitándole los criterios de selección de información para que pueda de utilidad en el estudio y en el desarrollo de las actividades planteadas en la asignatura.

- Incrementar el número de estudiantes que hacen uso de la tutoría.

Las actividades más relevantes que quedan recogidas en la guía docente de la asignatura y los recursos empleados se describirán a continuación teniendo en cuenta aspectos significativos desde el punto de vista metodológico. Debe tenerse en cuenta que las actividades no deben entenderse como elementos independientes sino que se conectan incluso en una misma sesión, por ejemplo, es habitual integrar sesiones de teoría de aula con prácticas de aula o incluso con prácticas de laboratorio.

\subsubsection{TA: Teoría de Aula.}

La clase magistral expositiva la consideramos muy útil si, desde un enfoque participativo, se da la oportunidad al alumnado de preguntar, reflexionar a partir de los contenidos teóricos, ejercicios o de preguntas abiertas. Esta metodología la empleamos fundamentalmente en las clases de TA ya que, aunque la materia de construcción tiene un carácter aplicado, se requiere de unos conocimientos básicos previos para poderse llevar a la práctica. Es importante obtener feedback de los alumnos a partir de preguntas abiertas ya que eso nos permitirá adoptar estrategias correctoras y reorientar aspectos en caso de ser necesarios. En este tipo de clases, la pizarra desempeña un papel fundamental, ya que permite la "construcción" del detalle, e incorporar su "ejecución" en un orden lógico (Ver Figura 1). Por otro lado, empleamos el proyector y el retroproyector, apuntes, publicaciones docentes, videos e imágenes de obras. Es habitual combinar la teoría de aula con prácticas de aula de corta duración. 


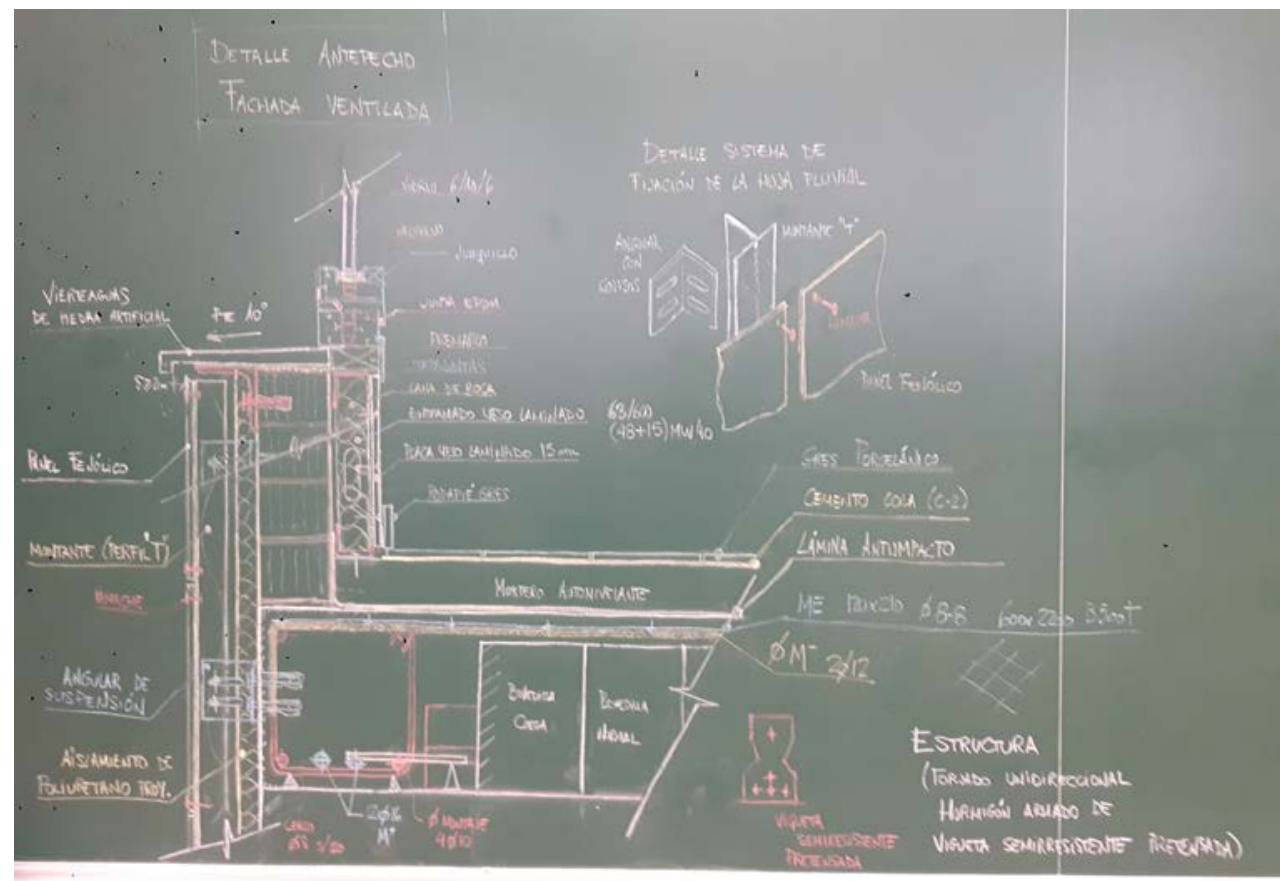

Fuente: Elaboración propia. J.M Gandía

Fig. 1 Representación en la pizarra de un detalle constructivo de CIII elaborado en clase (fachada ventilada)

\subsubsection{PA: Práctica de Aula.}

Las prácticas de aula son una de las actividades más relevantes ya que contribuyen a la adquisición de las competencias transversales y también de las específicas al estar conectadas directamente con las clases de teoría. Permiten consolidar los conocimientos, reforzar las ideas básicas, fomentar la capacidad de análisis y propiciar el acercamiento a situaciones reales. Los materiales y recursos empleados deben enfocarse a ese tipo de actividad. En este sentido, para el trabajo de las prácticas de aula se dispone de los "cuadernos de prácticas" que son documentos elaborados por el profesorado de la asignatura. Estos cuadernos son el soporte básico de muchos de los problemas que se plantean y son además un material que permite al alumnado trabajar de forma autónoma y recibir un feed-back en tutorías. 

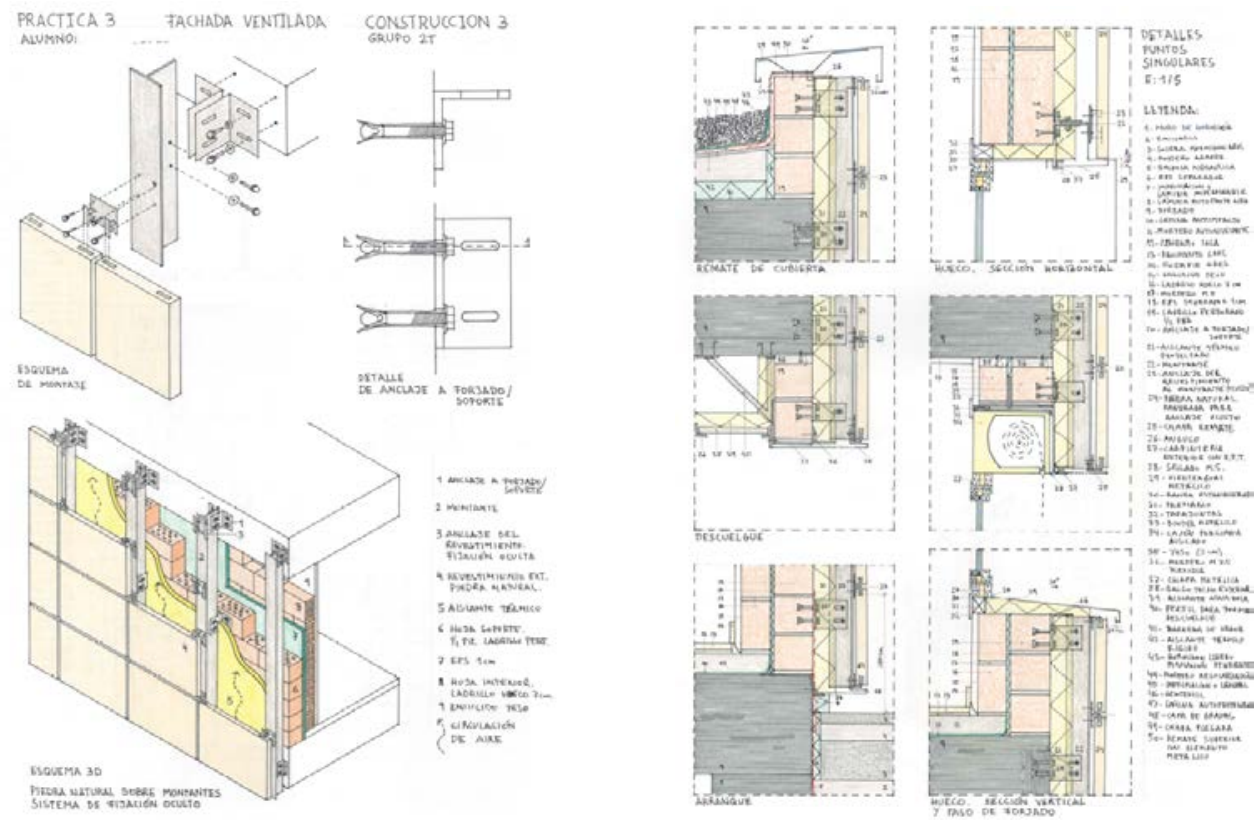

Alumno J. Saiz

Fig. 2 Práctica de Aula de CIII resuelta por un alumno del curso 20-21.

Se utilizan metodologías de aprendizaje basado en problemas (ABP) y métodos del caso mediante lo que denominamos visitas virtuales a obras. En las prácticas de aula, aparte de los cuadernos de prácticas y las lecciones publicadas, el recurso de las maquetas es clave de cara a fomentar la visión y comprensión espacial ya que todavía hay alumnos que tienen dificultades. Destacar también la metodología flip teaching que se ha implementado en los grupos de inglés (muy reducidos en cuanto a número de estudiantes) donde las clases son esencialmente prácticas y el alumnado dispone de un material específico que debe estudiar en casa,quedando la Teoría de Aula queda muy reducida en pro de la Práctica de Aula .

\subsubsection{PL: Práctica de Laboratorio}

Se desarrollan en el laboratorio de Construcción II-III Se hacen teniendo en cuenta unos objetivos muy concretos. El alumnado experimenta en primera persona ya que preferentemente tienen un carácter manipulativo. La finalidad es poner en contacto al alumno con materiales y analizar elementos constructivos, sistemas o tipologías. Se trabajan con recursos a escala y/o tamaño real y con materiales "reales" ya que los profesores hemos ido incorporando y renovando constantemente. También se dispone de muchos materiales disponibles gracias a la colaboración de empresas de referencia del sector que en determinados momentos son expuestos en la ETSIE. También se emplean maquetas realizadas por el alumnado de cursos previos de forma habitual en este tipo de actividades. 
Metodología y recursos didácticos claves el proceso de enseñanza-aprendizaje de las asignaturas de

Construcción II y III del Grado de Arq. Técnica - Methodology and key didactic resources used in teaching-learning process in the subjects of C II and III on Bachelor's Degree in Technical Architecture. En determinadas prácticas de laboratorio se realizan sesiones en colaboración con empresas del sector a modo de taller.

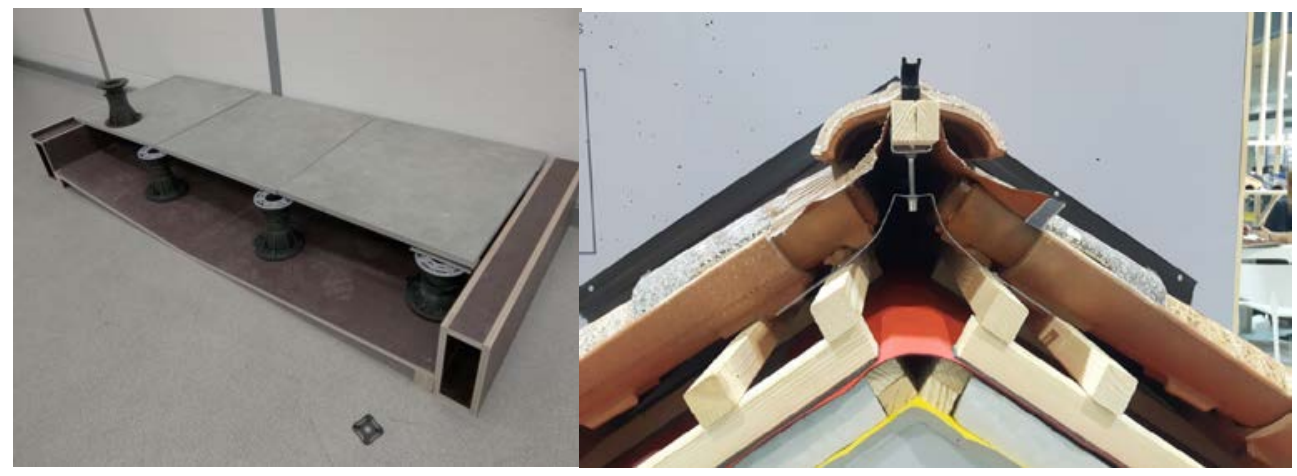

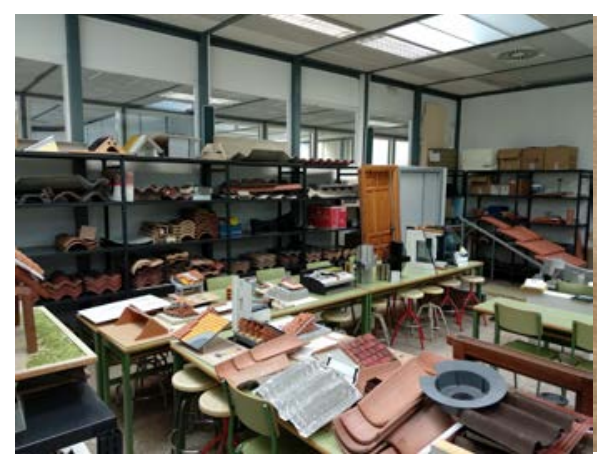

Fig. 3. Ejemplos de materiales y maquetas empleadas durante las prácticas de laboratorio

\subsubsection{PC: Práctica de Campo}

Como actividad tienen gran interés pedagógico, no hay que olvidar que la profesión de arquitecto técnico es eminentemente práctica, de hecho, en su origen nos encontramos con una profesión donde el aprendizaje se hacía de forma práctica. Las visitas a obras permiten que se pongan en relación conceptos, contenidos de distintas materias y que se contextualice en situación real, con sus interrelaciones constructivas, etc. Por otro lado, los talleres desarrollados en colaboración con empresas permiten profundizar en aspectos teóricoprácticos y ver "ejecuciones reales" que dan respuesta a numerosas cuestiones. Para las visitas a obra o talleres externos se rellena un formulario en la escuela y se proporciona un registro de participantes para tener cobertura con el seguro escolar. Por otro lado, cada vez es más frecuente que las grandes empresas de materiales tengan aulas de formación para aplicadores donde es posible orientar actividades a modo de taller. Un aspecto importante para nosotros es facilitar a los alumnos información de la visita/taller ya sea a partir de la plataforma PoliformaT de la UPV. 

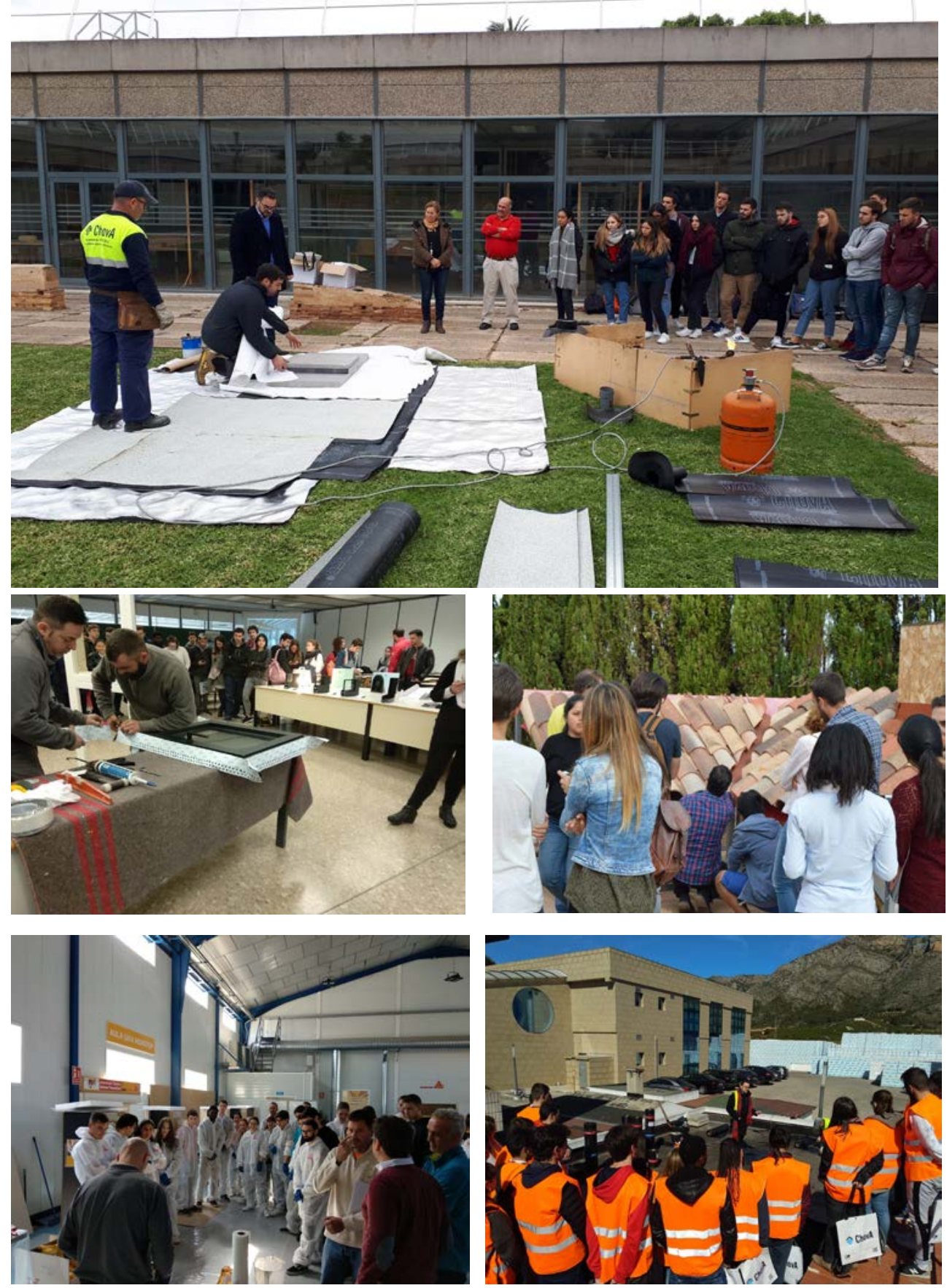

Fuente: Elaboración propia

Fig. 4. Talleres desarrollados con alumnos en colaboración con empresas. 
En la Figura 4 se incluyen algunos ejemplos de talleres realizados. Arriba el taller de instalación de laminas asfálticas realizado con la colaboración de Chova en la ETSIE; en medio a la izquierda: el taller de instalación de carpinterías para Passive House desarrollado en colaboración con VicenteTorres en la ETSIE; en medio a la derecha: el taller desarrollado en las instalaciones de Tejas Borja en Llíria sobre tejados ventilados y microventilados; abajo a la izquierda el taller realizado en colaboración con la empresa Sika en Quart de Poblet sobre instalación del sistema Sikatherm y abajo a la derecha el taller sobre puntos singulares en azoteas y visita a fábrica de XPS desaqrrollado en colaboración con Chova en Tavernes.
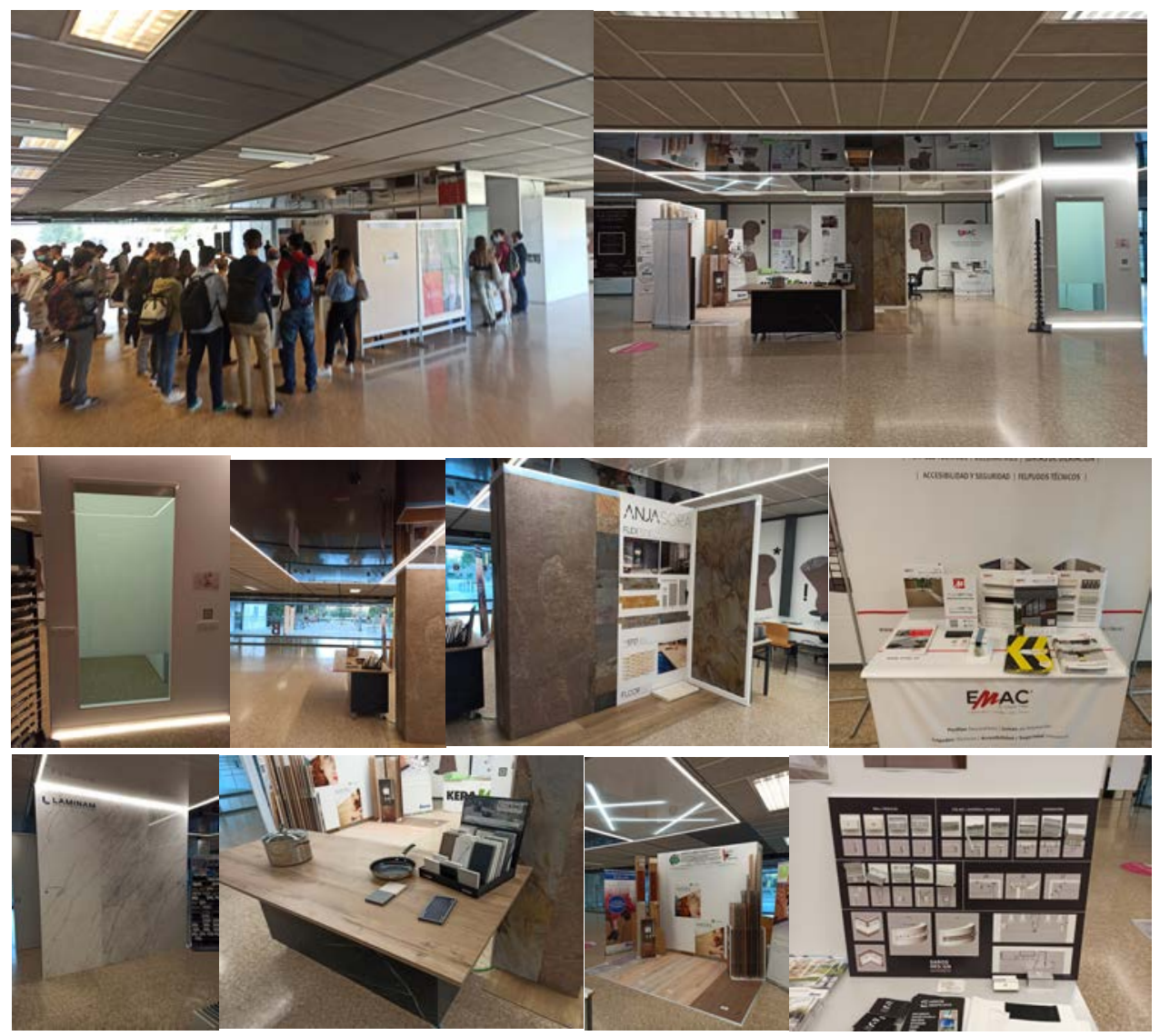

Fuente: Elaboración propia

Fig. 5. Exposición realizada en el Hall de la ETSIE sobre revestimientos y nuevos materiales.

Este año como novedad hemos introducido en la ETSIE la realización de una exposición (Figura 5) que permitiese a los alumnos profundizar en el bloque de revestimientos y ha sido 
muy interesante ya que se han expuesto materiales de varias empresas de forma temporal en el hall de la escuela y se han realizado varias charlas-seminario cortas en ese mismo espacio. Se han expuesto techos tensados, grandes formatos cerámicos, revestimientos pétreos naturales flexibles, soluciones de aislamiento acústico, nuevas soluciones de cocina, perfiles para pavimentos, adhesivos y materiales de rejuntado entre otros.

\subsubsection{TNP: Trabajo no presencial}

Como actividad tutorizada se plantea trabajar la metodología del aprendizaje por proyectos para que el alumno ponga en práctica los conocimientos y los aprendizajes adquiridos aplicándolos a una simulación de un proyecto real. Se busca la adquisición de competencias específicas y generales a través del aprendizaje cooperativo. Es una de las actividades donde más se fomenta la adquisición de habilidades de síntesis, de aplicación de los aprendizajes, de pensamiento práctico, de trabajo en equipo o de su creatividad por lo que potencian de una forma clara las competencias transversales. Los trabajos se desarrollarán en grupo, de 3 personas como máximo y el trabajo es tutorizado por el profesorado durante todo el semestre. Se les definen claramente los objetivos y se les orienta en los enfoques, la materialización, se resuelven las dudas y se asegurará que se cumple con los requisitos establecidos para su presentación.

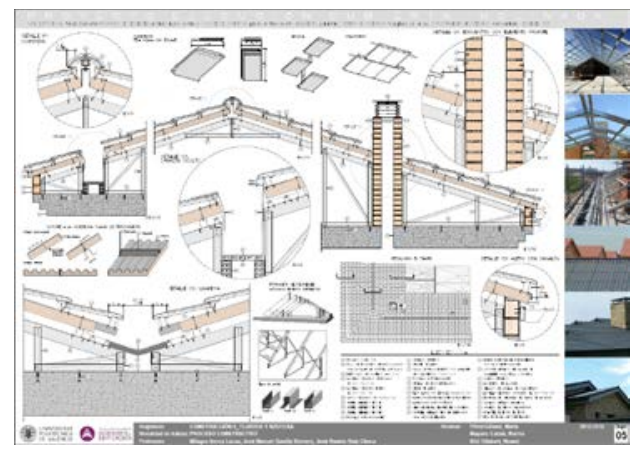

Fig. 6 Ejemplo de trabajo entregado por un grupo en la asignatura de CII en el curso 2018-2019.

\subsubsection{EVA: Actividades de evaluación}

Se realiza una evaluación continua multicriterio que permite evaluar al alumno teniendo en cuenta todas las actividades que llevan a cabodurante el curso (trabajos, examen, prácticas, problemas...) con lo que se consigue valorar de forma integral la asignatura. Los valores porcentuales de los actos de evaluación en Construcción II y III están reflejados en la guía docente. 
Metodología y recursos didácticos claves el proceso de enseñanza-aprendizaje de las asignaturas de

Construcción II y III del Grado de Arq. Técnica - Methodology and key didactic resources used in teaching-learning process in the subjects of C II and III on Bachelor's Degree in Technical Architecture.

\subsection{Análisis de la percepción del alumnado respecto al empleo de recursos didácticos}

A partir de las encuestas que periódicamente se emplean para evaluar el proceso de enseñanza-aprendizaje es posible tener un feedback sobre la percepción del alumnado respecto a la metodología y recursos ya que hay varias preguntas que evalúan esos aspectos.

En la Tabla 3 se incluyen los resultados de las encuestas de los últimos 5 años. Al analizar los resultados se puede observar que la valoración es alta.

Al preguntar si la recepción de la información al inicio de curso es adecuada, la valoración es es alta, con valor un promedio en las encuestas (s/10) de los últimos 5 años de 8.78 en Cll y 9.14 en CIII. En la pregunta que hace referencia a la metodología y actividades realizadas se obtienen promedios de 9.01 y 8.92. Cuando se les pregunta acerca de si los materiales recomendados a nivel de bibliografía y documentación són útiles para el aprendizaje se obtienen promedios de 8.51 y 8.76. En cuanto a la coherencia y utilidad de los recursos accesibles a través de la plataforma UPV (PoliformaT) la nota es de 8.33 y 8.67 .

Tabla 3. Resultados de las encuestas UPV durante los últimos 5 años en Cll y CIII referidas a metodologías y empleo de recursos

\begin{tabular}{|c|c|c|c|c|c|c|c|c|c|c|c|}
\hline & & \multicolumn{2}{|c|}{ 2016-2017 } & \multicolumn{2}{|c|}{ 2016-2017 } & \multicolumn{2}{|c|}{$2017-2018$} & \multicolumn{2}{|c|}{ 2018-2019 } & \multicolumn{2}{|c|}{ 2019-2020 } \\
\hline & & Nota s/10 & $\frac{\text { desviación }}{\text { típica }}$ & Nota $s / 10$ & $\frac{\text { desviación }}{\text { típica }}$ & Nota $s / 10$ & $\frac{\text { desviación }}{\text { típica }}$ & $\underline{\text { Nota } s / 11}$ & $\frac{\text { desviación }}{\text { típica }}$ & $\underline{\text { Nota } s / 10}$ & $\begin{array}{c}\frac{\text { desviación }}{\text { típica }} \\
\end{array}$ \\
\hline \multirow{4}{*}{ CII } & $\begin{array}{l}\text { AL INICIO DEL CURSO PROPORCIONA } \\
\text { INFORMACIÓN CLARA SOBRE LA } \\
\text { ASIGNATURA: OBJETIVOS, PROGRAMA, } \\
\text { METODOLOGÍA Y CRITERIOS DE } \\
\text { EVALUACIÓN }\end{array}$ & - & - & 8.33 & 1.86 & 9.22 & 1.92 & 9.04 & 1.61 & 8.56 & 1.42 \\
\hline & $\begin{array}{l}\text { LA METODOLOGÍA EMPLEADA Y LAS } \\
\text { ACTIVIDADES REALIZADAS EN LA } \\
\text { ASIGNATURA AYUDAN A APRENDER AL } \\
\text { ALUMNADO }\end{array}$ & $\cdot$ & - & 8.58 & 1.24 & 9.11 & 1.5 & 9.34 & 1.64 & 9.04 & 1.4 \\
\hline & $\begin{array}{l}\text { LOS MATERIALES UTILIZADOS Y/O } \\
\text { RECOMENDADOS (BIBLIOGRAFÍA, } \\
\text { DOCUMENTOS, RECURSOS DIDÁCTICOS, } \\
\text { ETC.) SON DE GRAN AYUDA PARA EL } \\
\text { APRENDIZAJE DE LA ASIGNATURA }\end{array}$ & - & - & 7.9 & 1.69 & 9.03 & 1.76 & 9.04 & 1.49 & 8.08 & 1.44 \\
\hline & $\begin{array}{l}\text { LOS MATERIALES DOCENTES HABILITADOS } \\
\text { ON-LINE (POLIFORMAT U OTROS ESPACIOS } \\
\text { ON-LINE OFICIALES EN LA UPV) POR ESTA } \\
\text { ASIGNATURA SON COHERENTES CON LOS } \\
\text { CONTENIDOS DEL PROGRAMA, } \\
\text { REPRESENTAN UNA IMPORTANTE AYUDA } \\
\text { PARA SU APRENDIZAJE Y PARA ALCANZAR } \\
\text { LOS OBJETIVOS DE LA ASIGNATURA. }\end{array}$ & - & - & 7.67 & 1.82 & 9.08 & 1.77 & 8.31 & 1.79 & 8.26 & 1.67 \\
\hline \multirow{4}{*}{ CIII } & $\begin{array}{l}\text { AL INICIO DEL CURSO PROPORCIONA } \\
\text { INFORMACIÓN CLARA SOBRE LA } \\
\text { ASIGNATURA: OBJETIVOS, PROGRAMA, } \\
\text { METODOLOGÍA Y CRITERIOS DE } \\
\text { EVALUACIÓN }\end{array}$ & 8.2 & 2.18 & 8.89 & 1.24 & 9.63 & 0.88 & 8.82 & 1.63 & 9.25 & 1.21 \\
\hline & $\begin{array}{l}\text { LA METODOLOGÍA EMPLEADA Y LAS } \\
\text { ACTIVIDADES REALIZADAS EN LA } \\
\text { ASIGNATURA AYUDAN A APRENDER AL } \\
\text { ALUMNADO }\end{array}$ & 8.9 & 2.13 & 8.75 & 1.5 & 9.88 & 0.54 & 8.82 & 2.03 & 8.25 & 2.65 \\
\hline & $\begin{array}{l}\text { LOS MATERIALES UTILIZADOS Y/O } \\
\text { RECOMENDADOS (BIBLIOGRAFÍA, } \\
\text { DOCUMENTOS, RECURSOS DIDÁCTICOS, } \\
\text { ETC.) SON DE GRAN AYUDA PARA EL } \\
\text { APRENDIZAJE DE LA ASIGNATURA }\end{array}$ & 7.7 & 2.64 & 8.06 & 2.13 & 9.31 & 1.48 & 8.55 & 2.36 & 9.15 & 1.59 \\
\hline & $\begin{array}{l}\text { LOS MATERIALES DOCENTES HABILITADOS } \\
\text { ON-LINE (POLIFORMAT U OTROS ESPACIOS } \\
\text { ON-LINE OFICIALES EN LA UPV) POR ESTA } \\
\text { ASIGNATURA SON COHERENTES CON LOS } \\
\text { CONTENIDOS DEL PROGRAMA, } \\
\text { REPRESENTAN UNA IMPORTANTE AYUDA } \\
\text { PARA SU APRENDIZAJE Y PARA ALCANZAR } \\
\text { LOS OBJETIVOS DE LA ASIGNATURA. }\end{array}$ & 7.6 & 2.4 & 8.82 & 1.94 & 8.82 & 2.42 & 7.79 & 2.41 & 9.25 & 1.69 \\
\hline
\end{tabular}

${ }^{*}$ En color azul: Periodo Covid 100\% Docencia online 
Dado que de este curso activo no se disponía de encuestas y al considerar necesario explorar de una forma más particularizada la percepción del alumnado sobre la utilidad de los recursos y sobre su adecuación al contexto de aprendizaje, se preparó una encuesta a través de la plataforma PolifoprmaT que ha sido respondida por 41 estudiantes de los 57 alumnos correspondientes a los grupos A y $\mathrm{T}$.

En ningún caso se ha obtenido una puntuación inferior a 8.5 puntos como se puede observar en la Tabla 4 donde incluimos los resultados derivados de la valoración de los estudiantes del curso 2020-2021.

Tabla 4. Resultados de las encuestas realizadas por los profesores en el curso 2020-2021

\begin{tabular}{|c|c|c|c|c|c|c|c|}
\hline & $\begin{array}{c}\text { Muy } \\
\text { insatisfecho } \\
\text { (1) }\end{array}$ & $\begin{array}{c}\text { Insatisfecho } \\
\text { (2) }\end{array}$ & Neutral (3) & $\begin{array}{l}\text { Satisfecho } \\
\text { (4) }\end{array}$ & $\begin{array}{c}\text { Muy } \\
\text { satisfecho (5) }\end{array}$ & Nota $s / 10$ & $\begin{array}{l}\text { desviación } \\
\text { típica }\end{array}$ \\
\hline $\begin{array}{l}\text { Considero que el uso de recursos materiales } \\
\text { desempeña (maquetas, muestras de material, } \\
\text { videos...) es clave en el proceso de enseñanza- } \\
\text { aprendizaje de las asignaturas de CII - CIII }\end{array}$ & 1 & 0 & 0 & 8 & 32 & 9.41 & 1.43 \\
\hline $\begin{array}{l}\text { Creo que asistir presencialmente a clase y el } \\
\text { contacto con los recursos es clave de cara a } \\
\text { abordar los procedimientos evaluables }\end{array}$ & 0 & 0 & 1 & 6 & 34 & 9.85 & 0.69 \\
\hline $\begin{array}{l}\text { El uso de maquetas en el aula es clave para el } \\
\text { aprendizaje de procedimientos y soluciones } \\
\text { constructivas }\end{array}$ & 0 & 0 & 1 & 17 & 23 & 9.07 & 1.10 \\
\hline $\begin{array}{l}\text { El uso de maquetas en el aula ha sido adecuado } \\
\text { para apoyar los contenidos }\end{array}$ & 1 & 0 & 1 & 5 & 34 & 9.51 & 0.98 \\
\hline $\begin{array}{l}\text { El uso de materiales de construcción en el aula es } \\
\text { clave para el aprendizaje de procedimientos y } \\
\text { soluciones constructivas }\end{array}$ & 0 & 0 & 1 & 11 & 29 & 9.37 & 1.04 \\
\hline $\begin{array}{l}\text { El uso de materiales de construcción en el aula ha } \\
\text { sido adecuado para apoyar los contenidos }\end{array}$ & 1 & 0 & 1 & 7 & 32 & 9.37 & 1.51 \\
\hline $\begin{array}{l}\text { El uso de fotografias y videos reales de obras en } \\
\text { ejecución en el aula es clave para el aprendizaje } \\
\text { de procedimientos y soluciones constructivas }\end{array}$ & 0 & 1 & 0 & 10 & 30 & 9.37 & 1.22 \\
\hline $\begin{array}{l}\text { El uso de fotografias y videos reales de obras en } \\
\text { ejecución en el aula ha sido adecuado para el } \\
\text { aprendizaje de procedimientos y soluciones } \\
\text { constructivas }\end{array}$ & 0 & 1 & 2 & 10 & 28 & 9.17 & 1.41 \\
\hline $\begin{array}{l}\text { Los detalles constructivos elaborados en la pizarra } \\
\text { son clave para el aprendizaje de procedimientos y } \\
\text { soluciones constructivas }\end{array}$ & 1 & 0 & 1 & 6 & 33 & 9.41 & 1.50 \\
\hline $\begin{array}{l}\text { El número y selección de los detalles elaborados } \\
\text { en la pizarra ha sido adecuado para el aprendizaje } \\
\text { de procedimientos y soluciones constructivas }\end{array}$ & 0 & 0 & 5 & 10 & 26 & 8.93 & 1.42 \\
\hline $\begin{array}{l}\text { Los apuntes de PoliformaT son un recurso clave } \\
\text { como apoyo a los apuntes de clase y de cara al } \\
\text { aprendizaje de la asignatura }\end{array}$ & 0 & 0 & 2 & 17 & 22 & 9.07 & 1.10 \\
\hline $\begin{array}{l}\text { Los apuntes de PoliformaT se pueden considerar } \\
\text { adecuados como complemento de los contenidos } \\
\text { desarrollados en las clases. Se consideran bien } \\
\text { estructurados }\end{array}$ & 0 & 2 & 6 & 12 & 21 & 8.54 & 1.79 \\
\hline $\begin{array}{l}\text { La realización de actividades como la Exposición } \\
\text { de Materiales con empresas del sector me ha } \\
\text { parecido interesante y motivadora. Es útil de cara } \\
\text { al aprendizaje }\end{array}$ & 0 & 0 & 3 & 16 & 22 & 8.93 & 1.27 \\
\hline $\begin{array}{l}\text { La realización de actividades como la Exposición } \\
\text { de Materiales con empresas del sector me ha } \\
\text { parecido interesante y motivadora. Es útil de cara } \\
\text { al aprendizaje }\end{array}$ & 0 & 1 & 3 & 10 & 27 & 9.07 & 1.49 \\
\hline $\begin{array}{l}\text { De forma general, se puede considerar que } \\
\text { metodológicamente y a nivel de recursos la } \\
\text { asignatura de Construcción III está bien } \\
\text { planificada }\end{array}$ & 0 & 0 & 3 & 8 & 30 & 9.17 & 1.67 \\
\hline
\end{tabular}




\section{Conclusiones}

En el presente trabajo se ha realizado una contextualización metodológica de las asignaturas de segundo curso de Construcción II y III en el grado de Arquitectura Técnica de la ETSIE. Se han realizado encuestas y, de forma general, se puede indicar que el grado de satisfacción por parte del alumnado es alto teniendo una percepción positiva sobre la utilidad de los recursos y los planteamientos metodológicos utilizados. Por otro lado, se ha podido observar que la base de recursos disponible ha permitido que en el periodo en el que la docencia pasó a la modalidad $100 \%$ no presencial por COVID se haya mantenido el nivel de satisfacción de los alumnos.

Aunque no se detectan carencias que puedan requerir de acciones correctivas a corto plazo si se considera al analizar en detalle los resultados, la conveniencia de planificar las acciones siguientes:

- Actualizar y mejorar los apuntes de Poliformat y de la bibliografía

- Analizar los detalles en pizarra con el fin de optimizar su selección y número

- Introducir herramientas de autoevaluación y tutoriales en determinadas actividades para reforzar el aprendizaje.

\section{Referencias}

BENITO, A., CRUZ, A. (2005). Nuevas claves para la docencia universitaria en el Espacio Europeo de Educación Superior. Madrid: Narcea.

GOÑI ZABALA, J.M. (2005). El Espacio Europeo de Educación Superior, un reto para la universidad. Barcelona: Octaedro / ICE Universidad de Barcelona

PERRENOUD , P (2007). Diez nuevas competencias Para enseñar. Invitación al viaje, Graõ, Colofón, México

POUMAY, M., TARDIF, J. ET GEORGES, F. (2017). Organiser la formation à partir des compétences : un pari gagnant pour l'apprentissage dans le supérieur. Louvain-laNeuve, Belgique : De Boeck Supérieur.

UNIVERSITAT POLITĖCNICA DE VALÈNCIA. Competencias Transversales <http://www.upv.es/contenidos/COMPTRAN/ > [Consulta: 20 de junio de 2021]

UNIVERSITAT POLITÈCNICA DE VALÈNCIA. Proyecto Europa < http://www.upv.es/europa/documen2.html> [Consulta: 26 de junio de 2021]

UNIVERSITAT POLITĖCNICA DE VALÈNCIA. Proyecto Europa <https://www.upv.es/entidades/ICE/info/638057normalc.html> [Consulta: 10 de junio de 2020]. 